\title{
AN ASSESSMENT OF THE DISPLACEMENT OF A CAM FOLLOWER USING REGULA FALSI METHOD
}

\author{
KOLAWOLE ADESOLA OLADEJO ${ }^{* 1}$, DARE ADERIBIGBE ADETAN ${ }^{1}$, ADEKOLA \\ OLAYINKA OKE ${ }^{1}$, RAHAMAN ABU ${ }^{2}$, GANIU AYOOLA LAMID ${ }^{1}$ \\ ${ }^{I}$ Department of Mechanical Engineering, Obafemi Awolowo University, Ile-Ife, Nigeria \\ ${ }^{2}$ Department of Mechanical Engineering, University of Ibadan, Nigeria
}

\begin{abstract}
Cam is a mechanical component that transforms circular motion to reciprocating motion by using mating component, called the follower. The principal aim of this work was to study and analyse the displacement of a cam-follower with Regula Falsi method and verify its input by using MATLAB and FORTRAN simulations. A study was conducted on angle of rotation and the displacement of the follower, which is equal to the radius of the cam given as transcendental equation to find the exact solution. The parameters such as initial guess, final guess, iteration counter and the desired displacement are involved in finding the angular displacement to the cam system in high speed rotation. The analysis was done using a computer programming that enables verification of the results obtained and ascertaining whether the inputs are correct or not for the displacement in cam follower system. The computer output showed results of the two data sets that yielded solutions and two that did not. The results revealed that the programme could be used to find the angular displacement corresponding to a given follower displacement for any cam; if the function CAMF is modified to include the appropriate radius function, $r(x)$. The results further revealed that at a halve cycle of a rotating cam, which is equivalent to $(x=3.142 \mathrm{rad})$, is a solution that would provide the desired displacement of the follower (opening and closing of valves).
\end{abstract}

Keywords: cam-follower, regula falsi, transcendental equation, cam angle, follower displacement

\section{INTRODUCTION}

In certain railroad applications where it is desirable to use passing railcar traffic to provide automatic mechanical action to a track-fixed system, developing a cam-follower mechanism can be among the best mechanical solutions, taking into account potential impact effects caused by the contact between the wheel and the trackfixed device. To design the cam-follower mechanism, the first decision to be made is determining the follower motion; since the main concerns are to minimize shock and wear on the follower and the driven device. In this case, cycloidal motion was selected. While most of the approaches in the field of cam design have been devoted to the synthesis of cam profiles, the major challenges in this case are to find an appropriate cam-follower mechanism and synthesize the follower surface profile to achieve cycloidal motion. We present a mechanism and simulate it using ADAMS software to prove the concept of our approach [1]. The different options involved in cam systems design such as types of cams and followers, number of applicable standard cam functions, divisions of follower motion-segments, and possible combinations of follower displacement profiles, among

\footnotetext{
* Corresponding author, email: wolesteady@yahoo.com

(C) 2021 Alma Mater Publishing House
} 
others, were critically studied to determine the structure and capabilities of a software package suited for extensive design analyses. The software was applied in the design of cam profiles with selected follower and cam functions and the results obtained were found comparable with those obtained from numerical methods and existing software packages [2]. A cam follower system was modelled in this work. The equation of motion and the eigen frequencies were recovered. The computation of the dynamic response was made by mean of combined implicit Newmark-Newton Raphson algorithm. Moreover, dynamic behaviour of the follower train was analysed in presence of an eccentricity in the camshaft and backlash between the follower rods and its guide [3].

The results validated the theorem in literature i.e. when prime circle radius is increased, the maximum cam pressure angle decreases which is desirable for cam design [4]. The results of this model compared reasonably with results obtained from DYNACAM. An Improved Regula Falsi (IRF) method based on classic Regula Falsi (RF) method is proposed in this paper. The method is verified on a number of test examples and numerical results confirm that the proposed method is very effective with respect to the classic Regula Falsi Method [5]. The dynamic force analysis determines the cam contact forces and values of kinematic parameters at which the design fails. The analytical calculation is done in excel sheet for both kinematic and dynamic analysis of cam follower system for any rotational speed and angle of rise [6]. The paper includes two simple design projects, generated by the software program to demonstrate important problems such as elevated pressure angle and undercutting that can occur in the design of cam mechanisms. The software tool was used in a lecture where positive feedback from students enrolled in the mechanisms course has encouraged the authors to continue the development of such educational software tools for other applications [7-8].

This work is aimed to develop a computer program for the purpose of analyzing and designing of Cam and Followers motion. The program was to be user-friendly and highly flexible in the analysis and design of cams and followers. To test the effectiveness of such, a computer model was developed for analysis and drafting of Cam and Follower motion on the platform of VBASIC. Users can input the required data, the program will then analyze the Cam Profile and display the numerical results in a list view exported to an Excel spread sheet for the graphical plotting. This paper also compared the results obtained from the developed application with manually analyzed and designed Cams [9]. An experimental apparatus that consists of a radial cam with an in-line knifeedge follower was developed for the study of the kinematics of valve-operating cam-follower mechanisms and the phenomenon of ignition in an internal combustion engine. The model incorporates a timer circuit, manual and automatic switches, and a micro switch circuit. It gives adequate room for data collection during experimentation. Experimentation with the apparatus produced results that are in very good agreement with analytical computations. It is also very stable and easy to use. In addition, the apparatus improved the student's learning apart from considerable cost reduction when compared to imported ones. This kind of experimental apparatus will be very useful in tertiary institutions in sub-Sahara Africa, where it has become very difficult to secure enough funds for the purpose of setting up standard laboratories for effective training of students of engineering and technology.

\section{MATERIALS AND METHODS}

Macaulay's method was adopted for the analyses of these beams in three types of loading application, concentrated load, linearly distributed load and applied moment. MATLAB is used in developing a model using the Macaulay's method in analyzing the beams. M-file codes are written using the derived equation to develop a user-friendly Graphic User Interface for the analysis of the beams. The results obtained from the developed model compared reasonably with analytical results [10]. The algorithm combines bisection with second and third order methods using derivatives estimated from objective functional values. Global convergence is ensured and the number of function evaluations is bounded by four times the number needed by bisection. Numerical comparisons with existing algorithms indicate the superiority of the new algorithm in all classes of problems [11]. The present paper unifies the notation and dimensionless analysis of the previous papers and compares both methods by applying them to the same design example. Furthermore, the analysis in the presence of friction is enhanced. Both methods are shown to yield accurately realized follower motions, even in the presence of a considerable amount of friction, despite the fact that friction is ignored during the design process [12-13].

The relative shape of the cam and follower define the displacement function of the mechanism, the displacement functions for radial cams are periodic functions consisting of sequences of dwell, rise and return segments. The displacement diagram are merely a plot of two different displacements, which are the distances travelled up or down by the follower and the angular distance rotated by the cam. The motion of the follower is the result of the 
program, just as a computer program, so is a cam. Thus, the system can be thought of as a mechanical information device. Accordingly, The goals of the designer is to build a program, establish the locus of the contact point between the cam and follower, produce the cam profile coordinate system, and fabricate the cam within an acceptable accuracy. After all the parts are assembled the performance of the cam-follower system is observed. Since the principle of cam follower is applicable to the opening and closing of valves in an internal combustion engine of an automobile, it has been assumed that at a halve cycle of a rotating cam which is equivalent to $\mathrm{x}=3.142 \mathrm{rad}$ is a solution that provides the desired displacement of the follower. MATLAB and FORTRAN were also used to verify the exact solution obtained.

\subsection{Theoretical background}

Cams turn rotary motion into reciprocating motions. Cams allow machines to apply pressure at particular moments in the cam's cycle. They are used in sewing machines to push the needle and thread through material in regular patterns. When many cams are threaded together on a camshaft, cams allow you to control the up and down movements of followers in particular sequences. Camshafts are used in car engines to open and close valves in sequence and control the flow of air, fuel and exhaust running through the engine. There are three cam exhibits: Circular cams, Pear-shaped cam and Heart-shaped cams [14-15]. Circular cams, also known as eccentric cams, are circle-shaped and fitted off-center onto the driving shaft. These cams move very smoothly and are used to generate a wave-like motion in the follower. They are often used in mechanical toys to produce a smooth, recurring motion. In each toy gear, a pencil tip takes the place of the axle, and the outer frame of the toy acts as a follower. Because the frame is rigid, it does not move-instead it forces the toy gear's free axle to move up and down as the gear rotates. The pencil point at the axle draws intricate spirals. The closer the axle is to the edge, the more eccentric the pattern becomes. Pear-shaped cams are actually egg shaped. The follower is level for most of the cam's rotation, and then is suddenly pushed up at regular intervals. These are used in car engines to control the flow of fuel and exhaust in the engine. Heart-shaped cams allow the follower to move at a constant velocity and therefore provide a very steady motion. They are used in sewing machines to wind thread onto the bobbin.

Transcendental equations arise in a large number of science and engineering problems. In falsi position method, the study of exact solutions to these non-linear equations play a very germane role, as it provide much information about the physical models it describes. In this paper, we apply the regula falsi method to get the angular rotation of the cam from the cam radius which is equivalent to the desired displacement of the follower as shown in the Figure 1. The radius " $r$ " of the cam as a function of the cam angle " $x$ " is of the form equation (1).

$$
r(x)=0.55+0.5 e^{\left(\frac{-x}{2 \pi}\right)} \sin (x), 0 \leq x \geq 2 \pi
$$

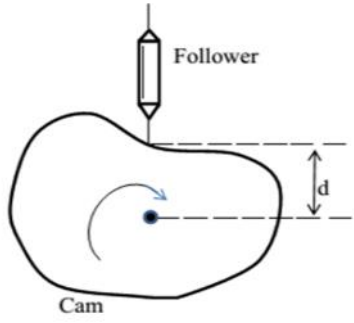

(a)

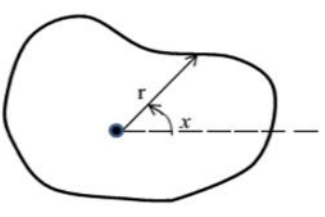

(b)

$\begin{array}{ll}\text { (a) Cam and follower. } & \text { (b) Rotation angle, } x \text {, and radius, } r \text {. }\end{array}$

Fig. 1. Displacement of a cam follower.

\subsection{Description of the regula falsi method}

Here, a brief explanation of the Regula Falsi Method for finding the exact angular rotation of the cam. Consider a transcendental equation of the form equatin (2).

$$
f(x)=0
$$


where $f(a)$ and $f(b)$ are of opposite signs. Also let $a<b$.

The graph $r=f(x)$ will meet the $\mathrm{x}$ - axis at some point between $A(a), f(a))$ and $B(b, f(b))$. The equation of the chord joining the two points $A(a, f(a))$ and $B(b, f(b))$ is equation (3).

$$
\frac{r-f(a)}{x-a}=\frac{f(a)-f(b)}{x-b}
$$

where the $\mathrm{x}$-coordinate of the point of intersection of this chord with the $\mathrm{x}$-axis gives an approximate value for the $f(x)=0$. Taking $r=0$ in the chord equation, we get equations (3)-(5).

$$
\begin{gathered}
\frac{-f(a)}{x-a}=\frac{f(a)-f(b)}{x-b} \\
x[f(a)-f(b)]-a f(a)+a f(b)=-a f(a)+b f(b) \\
x[f(a)-f(b)]=b f(b)-a f(b)
\end{gathered}
$$

This $x_{1}$ gives an approximate value of the root $f(x)=0 .\left(a<x_{1}<b\right)$. Now $f\left(x_{1}\right)$ and $\mathrm{f}($ a)are of opposite signs or $\mathrm{f}\left(\mathrm{x}_{1}\right)$ and $\mathrm{f}(\mathrm{b})$ are opposite signs. If $f\left(x_{1}\right) f(a)<0$. Then $x_{l}$ lies between $x_{1}$ and $a$ equation (7).

$$
x_{1}=\frac{a f\left(x_{1}\right)-x_{1} f(b)}{f\left(x_{1}\right)-f(a)}
$$

This process of calculation of $\left(x_{2}, x_{3}, x_{4} \ldots\right)$ is continued till any two successive values are equal and subsequently we get the solution of the given equation. The performance of the regula falsi was evaluated on the basis of its approximate error as equation (8):

$$
\varepsilon_{a}=\left|\frac{x_{\text {new }}-x_{\text {old }}}{x_{\text {new }}}\right| \times 100 \%
$$

Steps:

Find $a$ and $b$ in which $f(a)$ and $f(b)$ are opposite signs for the given equation using trial and error method. Therefore root lies between $a$ and $b$ if $f(a)$ is very close to zero select and compute $x_{1}$ by using the following formula (9):

$$
x_{1}=\frac{a f(b)-b f(a)}{f(b)-f(a)}
$$

If $f\left(x_{1}\right) f(a)<0$. Then root lies between $x_{1}$ and $a$. Compute $x_{2}$ by using the following formula (10):

$$
x_{2}=\frac{a f\left(x_{1}\right)-x f(b)}{f\left(x_{1}\right)-f(a)}
$$

Calculate the values of $\left(x_{3}, x_{4}, x_{5}, \ldots\right)$ by using the above formula until any two successive values are equal and subsequently we get the solution of the given equation.

When $D=0.50 \mathrm{~mm}$, the equation becomes equation (11):

$$
f(x)=0.5 e^{(-x / 2 \pi)} \sin (x)=0
$$


This means that in order to get a reasonable angle of rotation between the interval $[0,2 \pi]$, the equation needs to be modified.

The first iteration is:

$$
\begin{aligned}
& x_{r}=\frac{0.7000(-0.147016)-3.7000(0.288149)}{-0.147016-0.288149}=2.686480 \\
& f(0.7000) \times f(2.686480)=0.288149(0.1433149)=0.041297
\end{aligned}
$$

Therefore, the root is in the first interval, and the lower guess is redefined as $x_{L}=2.686480$.

The second iteration is:

$$
\begin{gathered}
x_{r}=\frac{2686480(-0.147016)-3.7000(0.143318)}{0.147016-0.143318}=3.186788 \\
\varepsilon_{a}=\left|\frac{3.186788-2.686480}{3.186788}\right| \times 100 \%=15.70 \% \\
f(2.686480) \times f(3.186788)=0.143326(-0.013603)=-0.001950
\end{gathered}
$$

Therefore, the root is in the first interval and the upper guess is redefined as $x_{\mathrm{R}}=3.186788$. The remainders of the iterations are displayed in Table 1 and Table 2. The angular displacement of the cam when $D$ is equal to 0.70 $\mathrm{mm}, 0.75 \mathrm{~mm}, 1.00 \mathrm{~mm}$ etc. shall be explicitly solved later with regula falsi algorithm using MATLAB and FORTRAN.

Table 1. Computation of regula falsi method.

\begin{tabular}{cccccccc}
\hline$i$ & $x_{L}$ & $f\left(x_{L}\right)$ & $x_{R}$ & $f\left(x_{R}\right)$ & $x_{r}$ & $f\left(x_{r}\right)$ & $\left|\varepsilon_{a}\right| \%$ \\
\hline 1 & 0.700000 & 0.288149 & 3.700000 & -0.147016 & 2.686480 & 0.143318 & \\
2 & 2.686480 & 0.143318 & 3.700000 & -0.147016 & 3.186788 & -0.013603 & 15.70 \\
3 & 2.686480 & 0.143318 & 3.186788 & -0.013603 & 3.143416 & -0.000553 & 1.380 \\
4 & 2.686480 & 0.143318 & 3.143416 & -0.000553 & 3.141655 & -0.000019 & 0.056 \\
5 & 2.686480 & 0.143318 & 3.141655 & -0.000019 & 3.141596 & -0.000001 & 0.002 \\
\hline
\end{tabular}

Table 2. Corresponding cam radius with various follower displacements.

Follower Displacement, D(mm)

0.50
0.70
0.75
1.00
1.50
2.00

0.50

0.70

0.75

1.50

2.00
Cam Radius, $\mathrm{R}(\mathrm{x}), \mathrm{Rad}$

$0.5 \mathrm{e}^{(-\mathrm{x} / 2 \pi)} \sin (\phi)-0.00$

$0.5 \mathrm{e}^{(-\mathrm{x} / 2 \pi)} \sin (\phi)-0.20$

$0.5 \mathrm{e}^{(-\mathrm{x} / 2 \pi)} \sin (\phi)-0.25$

$0.5 \mathrm{e}^{(-\mathrm{x} / 2 \pi)} \sin (\phi)-0.50$

$0.5 \mathrm{e}^{(-\mathrm{x} / 2 \pi)} \sin (\phi)-1.00$

$0.5 \mathrm{e}^{(-\mathrm{x} / 2 \pi)} \sin (\phi)-1.50$

\subsection{Performance of regula falsi method to the present problem}

The performance of the regula falsi was evaluated on the basis of its approximate error. Figure 2 present the flow chart for regula falsi algorithm equation (12).

$$
\varepsilon_{a}=\left|\frac{x_{\text {new }}-x_{\text {old }}}{x_{\text {new }}}\right| \times 100 \%
$$




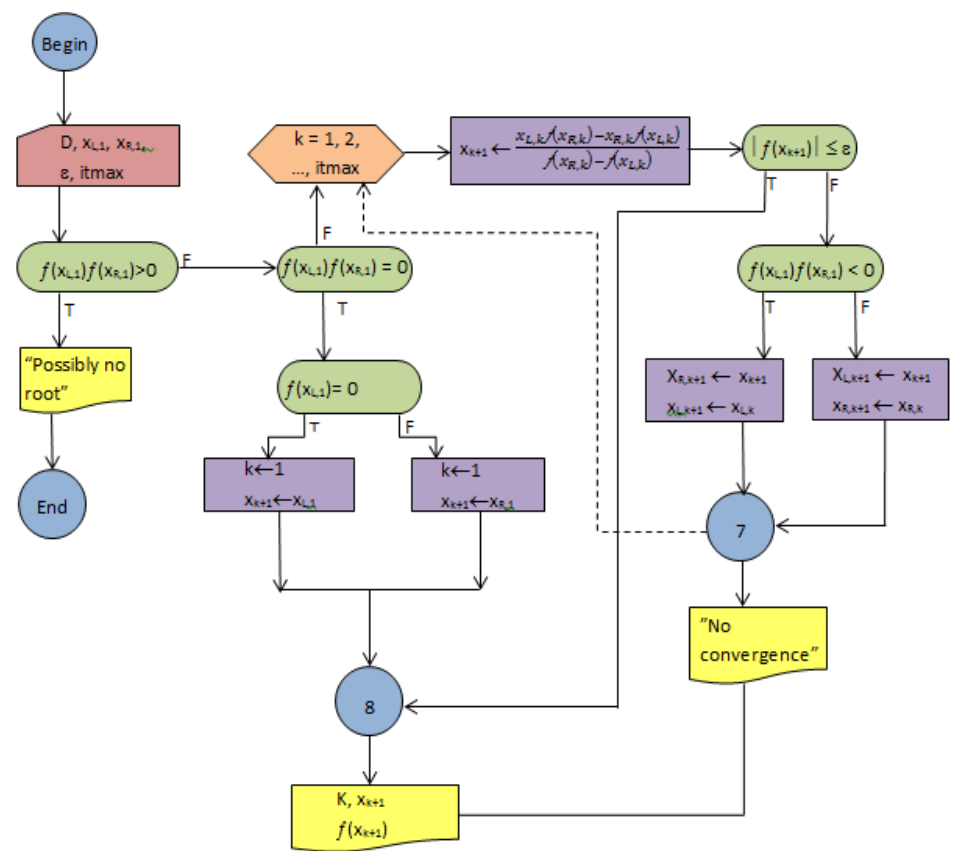

Fig. 2. Flow chart for regula falsi algorithm.

\subsection{Model development}

In this section, we apply the regula falsi method to get the exact value of angular rotation of a cam in one revolution, from the equation (1) which is the radius of the cam equation (13).

$$
r(x)=0.55+0.5 e^{\left(\frac{-x}{2 \pi}\right)} \sin (x)
$$

Consider the rotating cam with the follower shown in Figure 1. Let $\mathrm{d}(\mathrm{mm})$ be the displacement of the follower tip measured from the center of rotation of the cam. The radius of the cam $r(\mathrm{~mm})$, measured from the center of rotation angle, $x$ (radians). In order to use regula falsi algorithm to find a rotation angle in the angular interval $\left[x_{L}, x_{R}\right]$ for which the follower displacement $d(x)$ is equal to $D$. The convergence criterion for stopping the iterative computation should be equation (14):

$$
|d(x)-D| \leq \varepsilon
$$

For a given angle of rotation $x, d(x)=r(x)$. The angle $x$ that produces the desired displacement $D$ is the solution of the equation (15).

$$
f(x)=r(x)-D=0.5+0.5 e^{(-x / 2 \pi)} \sin (x)-D=0
$$

Convergence criterion $|f(x)| \leq \varepsilon$. The angular radius of the cam $x$ varies from 0 to $2 \pi \mathrm{rad}$. In order to get a reasonable angle of rotation between the intervals $[0,2 \pi]$, the equation just need to be modified.

\section{RESULTS AND DISCUSSION}

The results of the performance evaluation of the regula falsi algorithm using both the MATLAB and FORTRAN Simulink are presented in Figures 3, 4, 5, 6, 7 and 8. Also, Figure 9 shows the follower displacement, $d$, as a function of angle of rotation, $x$. Approximate Error $\left(\varepsilon_{\mathrm{a}}\right)$. From Figures 3, 4, 5, 6, 7 and 8, the approximate error $\left(\varepsilon_{\mathrm{a}}\right)$ of regula falsi algorithm is $\left|\frac{3.141596-3.141655}{3.141596}\right| \times 100 \%, \varepsilon_{a}=0.002 \%$. Therefore, after $90^{\text {th }}$ iterations, an 
estimated value of 3.141896 with an approximate error of $0.002 \%$ which is below the convergence criterion was obtained. From Figure 9, it shows that the graph touches the angular rotation ' $x$ ' at 3.1418 radian (Approximate).

Case 1: When, $D=0.50 \mathrm{~mm}$, the radius of the cam becomes equation (16):

$$
r(x)=0.5 e^{(-x / 2 \pi)} \sin (x)
$$

This means that in order to get a reasonable angle of rotation between the intervals $[0,2 \pi]$, the equation needs to be modified. Here, $x_{L}$ be lower guess, $x_{R}$ be upper guess, $f\left(x_{L}\right)$ be function of my lower guess, $f\left(x_{R}\right)$ be function of my upper guess, If $x_{L}=0.7000$, and $x_{R}=3.7000$, then $f\left(x_{L}\right)=0.288149$, and $f\left(x_{R}\right)=-0.147016$.

Therefore $f\left(x_{L}\right) \times f\left(x_{R}\right)<0$. Since $f\left(x_{L}\right)$ and $f\left(x_{R}\right)$ are of opposite sign, it means that the root exist on the interval $\left[x_{L}, x_{R}\right]$, from equation (9) the regula falsi algorithm can now be implemented iteratively and the result is shown in Figure 3 ( $a, b, c$ and d).

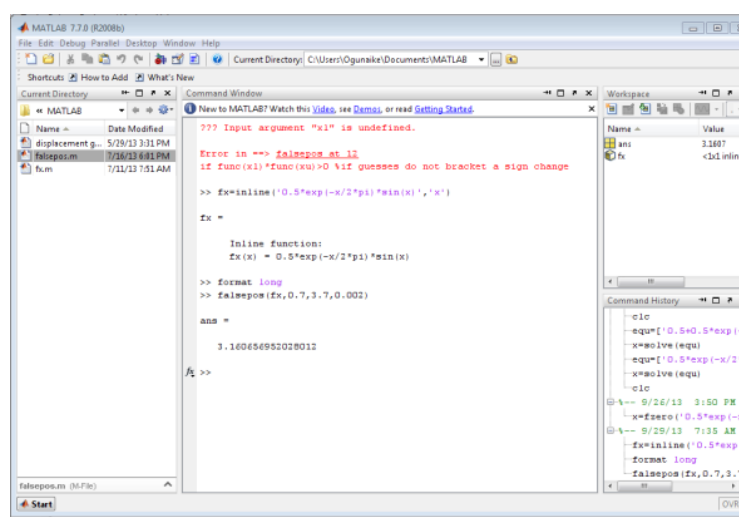

(a)

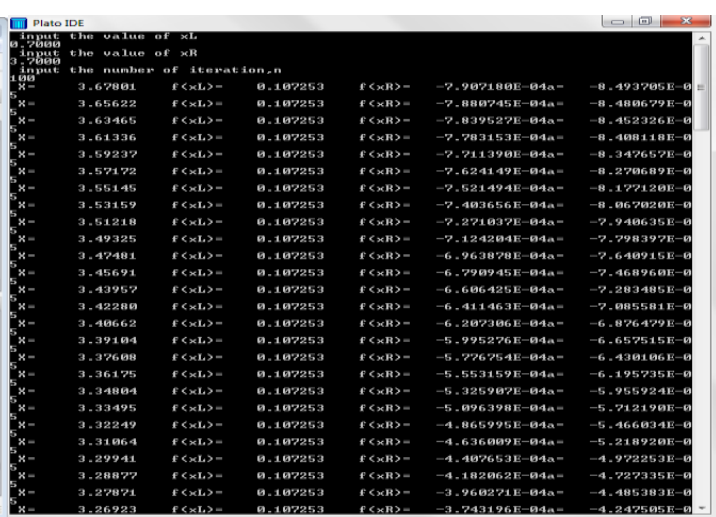

(b)

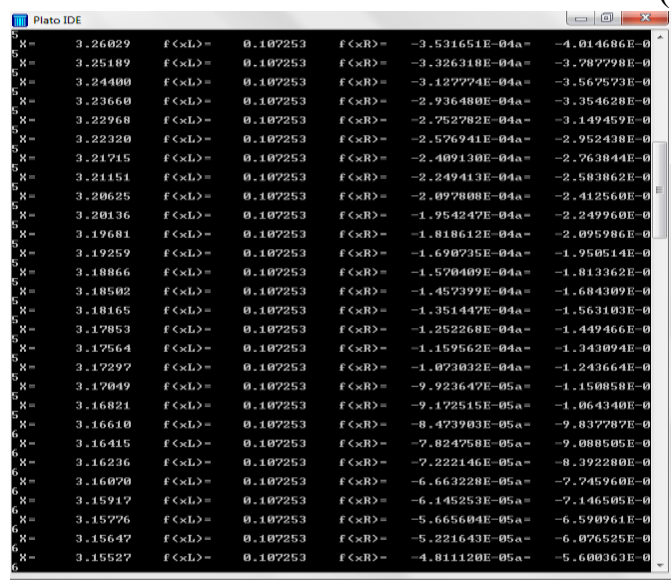

(c)

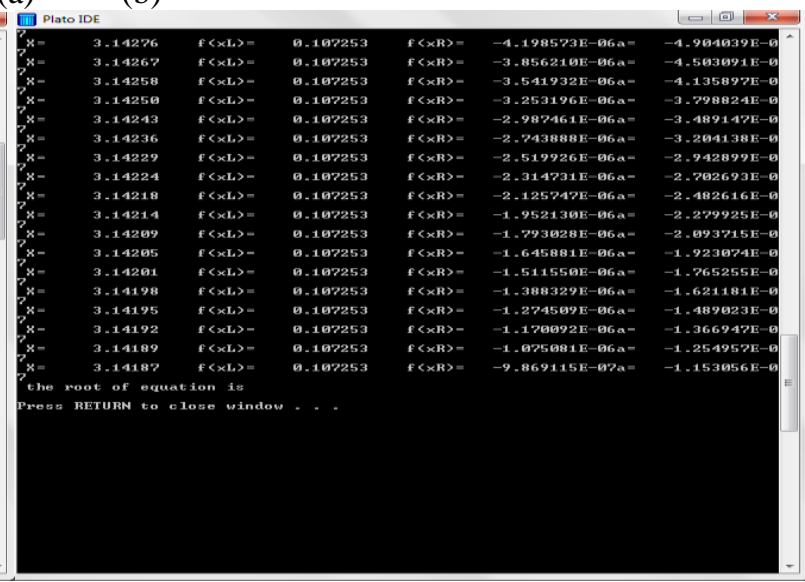

(d)

Fig. 3. Angle of rotation for $0.50 \mathrm{~mm}$ follower displacement: a), b), c), d) results.

Case 2: When, $\mathrm{D}=0.70 \mathrm{~mm}$, the radius of the cam becomes, equation (17):

$$
r(x)=0.5 e^{(-x / 2 \pi)} \sin (x)-0.20
$$

Since $f(x L)$ and $f(x R)$ are of opposite sign, it means that the root exist on the interval $[x L, x R]$, from equation (9) the regula falsi algorithm can now be implemented iteratively and the result is shown in Figure 4 (a and b). 


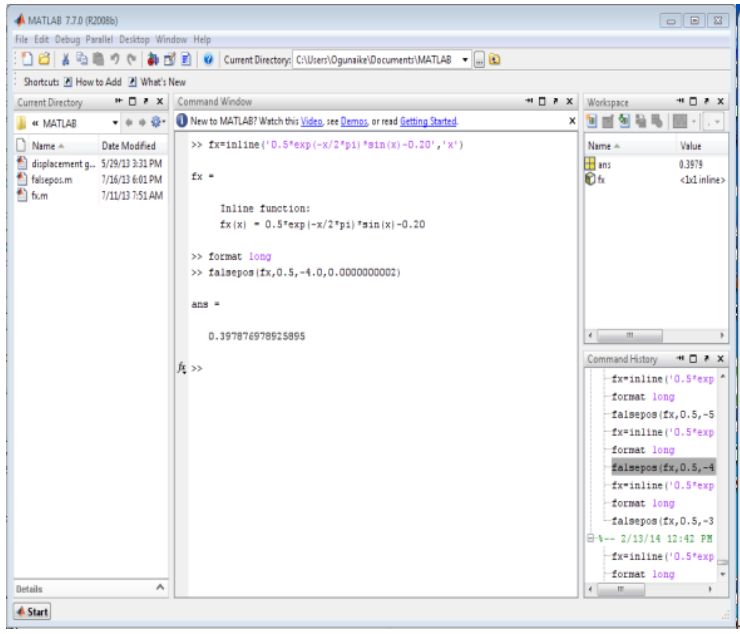

(a)

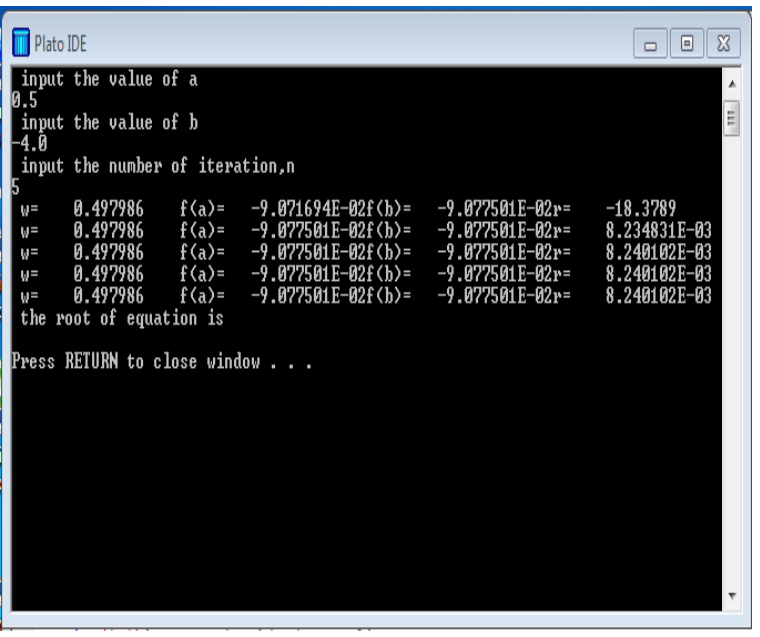

(b)

Fig. 4. Angle of rotation for $0.70 \mathrm{~mm}$ follower displacement: a), and b) are results.

Case 3: When, $D=0.75 \mathrm{~mm}$, the radius of the cam becomes equation (18):

$$
r(x)=0.5 e^{(-x / 2 \pi)} \sin (x)-0.25
$$

Since $f(x L)$ and $f(x R)$ are of opposite sign, it means that the root exist on the interval $[x L, x R]$, from equation (9) the regula falsi algorithm can now be implemented iteratively and the result is shown in Figure 5 (a and b).

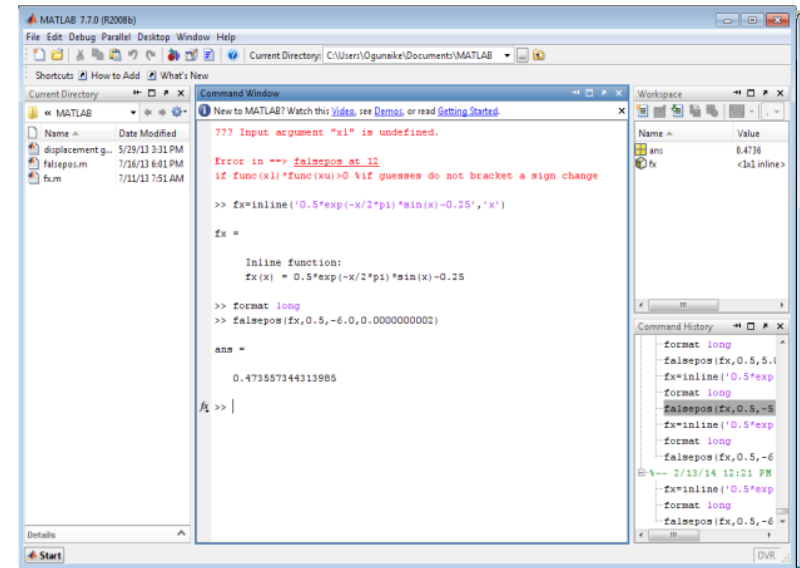

(a)

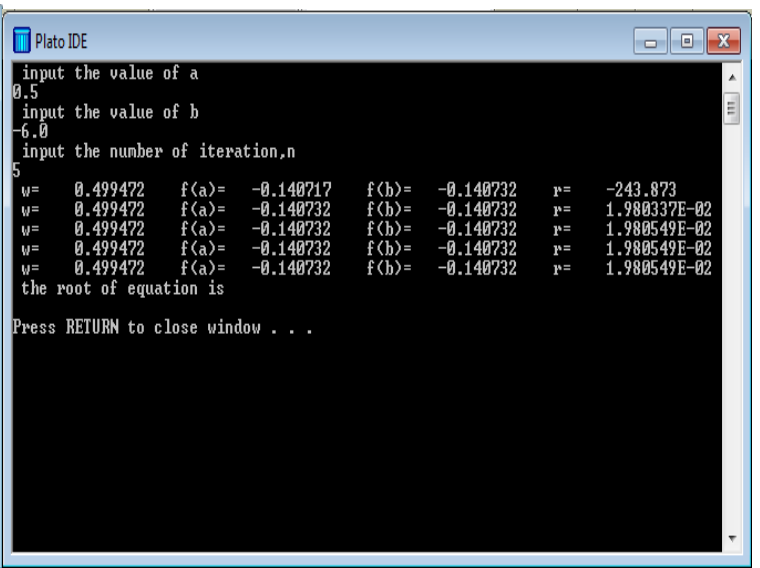

(b)

Fig. 5. Angle of rotation for $0.75 \mathrm{~mm}$ follower displacement: a), and b) are results.

Case 4: When, $D=1.00 \mathrm{~mm}$, the radius of the cam becomes equation (19):

$$
r(x)=0.5 e^{(-x / 2 \pi)} \sin (x)-0.50
$$

Since $f(x L)$ and $f(x R)$ are of opposite sign, it means that the root exist on the interval $[x L, x R]$, from equation (9) the regula falsi algorithm can now be implemented iteratively and the result is shown in Figure 6 (a and b). 


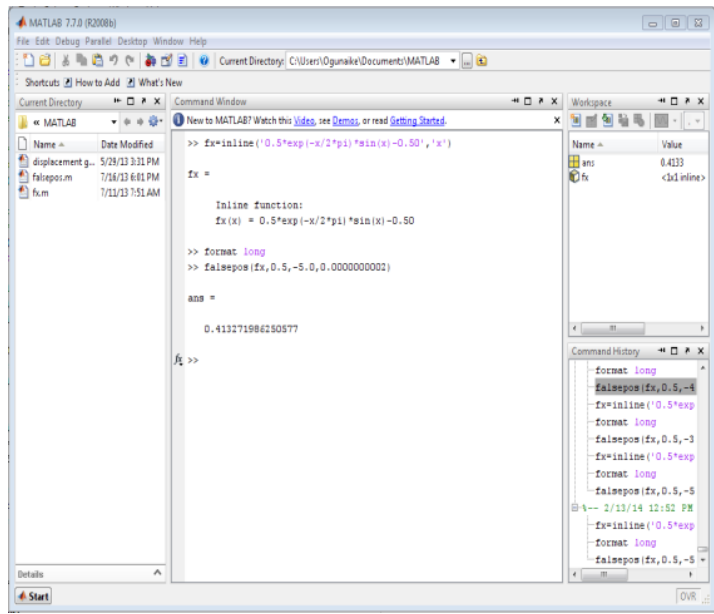

(a)

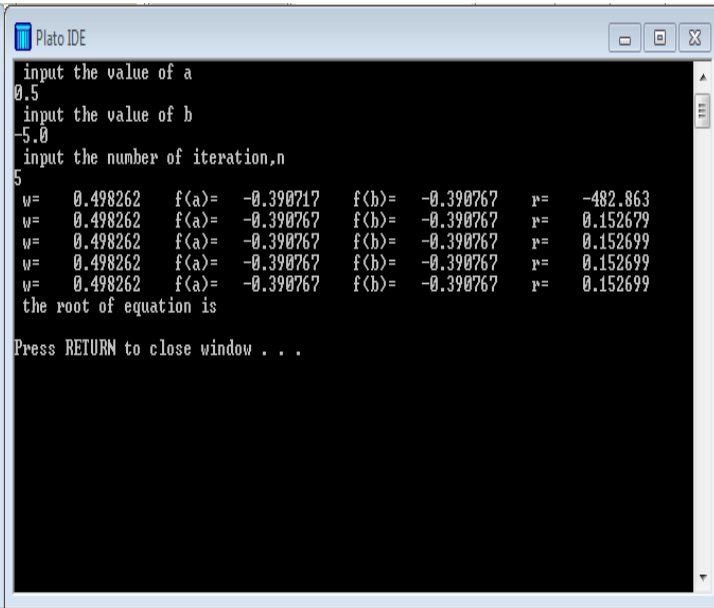

(b)

Fig. 6. Angle of rotation for $1.00 \mathrm{~mm}$ follower displacement: a), and b) are results.

Case 5: When, $D=1.50 \mathrm{~mm}$, the radius of the cam becomes equation (20):

$$
r(x)=0.5 e^{(-x / 2 \pi)} \sin (x)-1.00
$$

Since $f(x L)$ and $f(x R)$ are of opposite sign, it means that the root exist on the interval $[x L, x R]$, from equation (9) the regula falsi algorithm can now be implemented iteratively and the result is shown in Figure 7 (a and b).

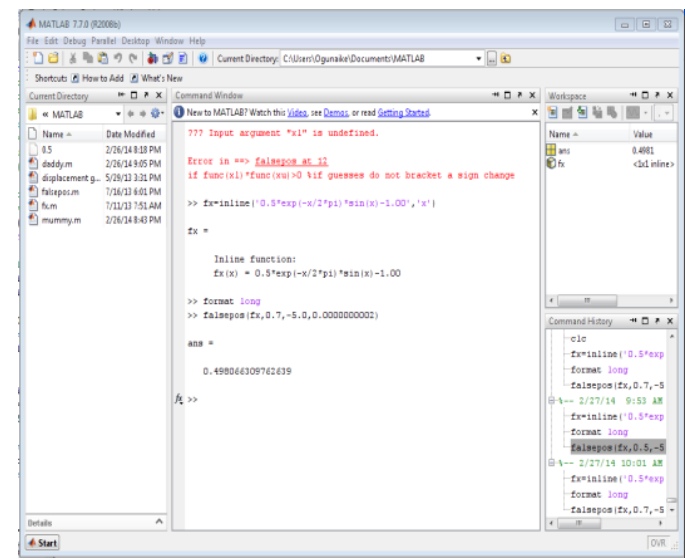

(a)

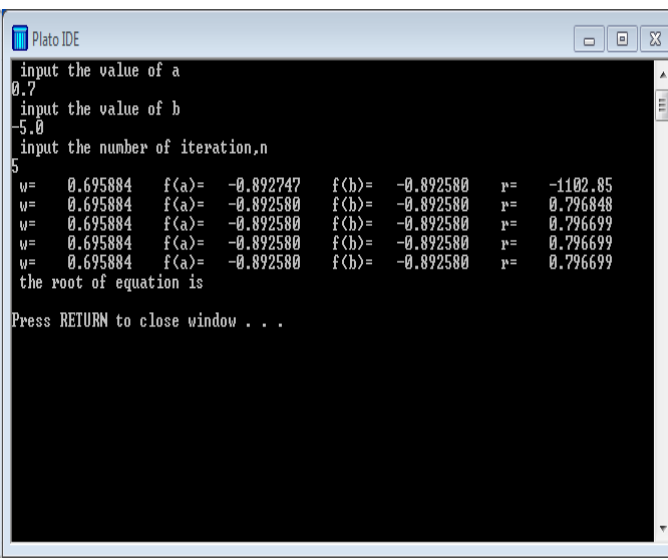

(b)

Fig. 7. Angle of rotation for $1.50 \mathrm{~mm}$ follower displacement: a), and b) are results.

Case 6: When, $\mathrm{D}=2.00 \mathrm{~mm}$, the radius of the cam becomes equation (21):

$$
r(x)=0.5 e^{(-x / 2 \pi)} \sin (x)-1.50
$$

Since $f(x L)$ and $f(x R)$ are of opposite sign, it means that the root exist on the interval $[x L, x R]$, from equation (9) the regula falsi algorithm can now be implemented iteratively as shown in Figure 8 (a and b).

The computer output showed result for six data sets that yielded solutions (The exact solution for the first data set is $\mathrm{x}=\pi$.), which generate the desired displacement of the cam follower. The programs can be used to find the angular displacement corresponding to a given follower displacement for any cam; only the function CAMF needs to be modified to include the appropriate radius function, $r(x)$. The graph for Case 1, Case 2, Case 3, Case 4, Case 5 and Case 6 for selected values of ' $d$ ' are presented in Figure 9. 


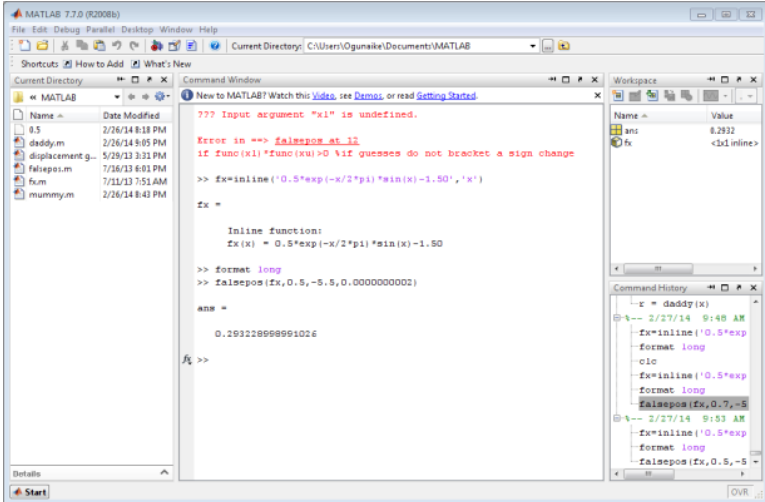

(a)

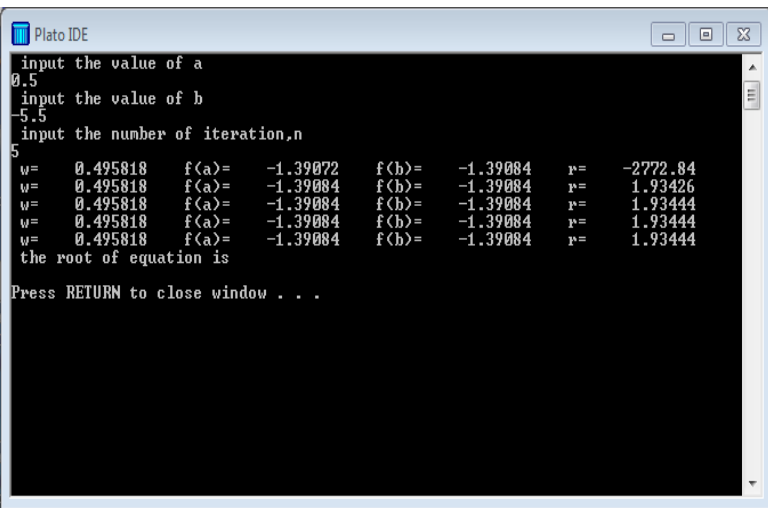

(b)

Fig. 8. Angle of rotation for $2.00 \mathrm{~mm}$ follower displacement: a), and b) are results.

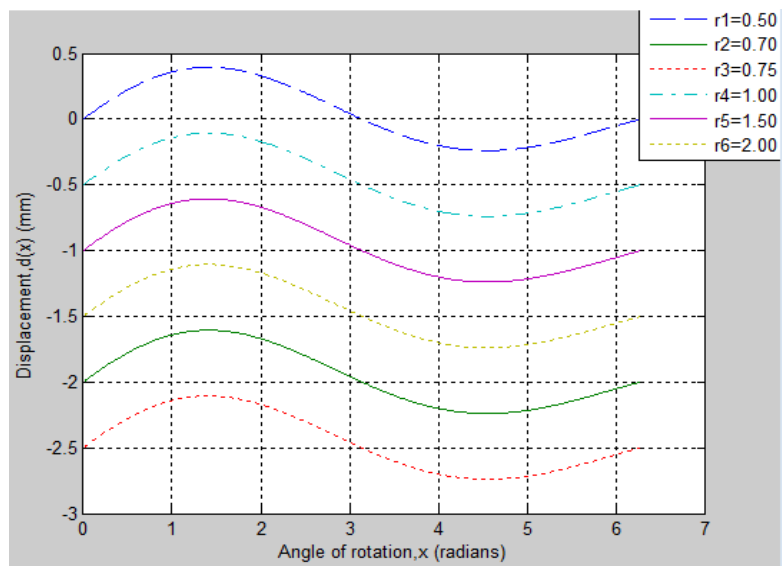

Fig. 9. Actual variation of angle of rotation with displacement for different values of cam radius.

\section{CONCLUSION}

The angular displacement solution for the transcendental equation of a cam radius have been obtained using the regula falsi method. Since the principle of cam follower is applicable to the opening and closing of valves in an internal combustion engine of an automobile, it is assumed that halve cycle of a rotating cam which is equivalent to $(\mathrm{x}=3.142 \mathrm{rad})$ is a solution that provide the desired displacement of the follower (opening and closing of valves). MATLAB and FORTRAN were also used to verify the exact solution obtained. Instead of having to go through a tedious process of plotting something by hand or at some point where analytical solutions are not possible, MATLAB generate a nice plot and solution that is desired. Therefore, the performance trend of regula falsi method has made it an important and viable alternative to other numerical methods for a broader range of applications.

$\begin{array}{ll}\text { NOTATION } & \\ \text { FUNT } & \text { Function that compute the value of } \boldsymbol{r}(\boldsymbol{x}), \mathbf{m m} \\ D \text { ord } & \text { Cam follower displacement, } D, \mathrm{~mm} \\ \text { EPS } & \text { Tolerance for convergence criterion, } \varepsilon_{a}, \mathrm{~mm} \\ \text { ITER } & \text { Iteration counter, } n \\ \text { ITMAX } & \text { Maximum number of iterations permitted, itmax } \\ x_{L}, x_{U}, x_{r} & x_{L}, x_{U}, \text { and } x_{r+1}, \text { rad } \\ r & \text { Radius of the cam, (mm) } \\ x & \text { Angular rotation of a cam, (rad) } \\ a & \text { Initial guess } \\ b & \text { Final guess } \\ \boldsymbol{f x}_{L}, \boldsymbol{f} x_{U}, \boldsymbol{f} x_{\boldsymbol{r}} & \boldsymbol{f}\left(\boldsymbol{x}_{\boldsymbol{L}}, \boldsymbol{n}\right), \boldsymbol{f}\left(\boldsymbol{x}_{\boldsymbol{u}}, \boldsymbol{n}\right), \text { and }\left(\boldsymbol{x}_{\boldsymbol{n}+1}\right), \mathbf{m m}\end{array}$




\section{ACKNOWLEDGEMENT}

The authors acknowledge the facilities and support of the Modeling laboratory of Department of Mechanical Engineering, Obafemi Awolowo University, Ile-Ife, Nigeria.

\section{REFERENCES}

[1] Abolfazl, P., Carl, A.N., A case study of designing a cam-follower mechanism with cycloidal motion, 13th World Congress in Mechanism and Machine Science, Guanajuato, México, June, 2011, p. 19-25.

[2] Simolowo, O.E., Bamiro, O.A., Roller-cam systems design: development of a profile analysis software, Pacific Journal of Science and Technology, vol. 10, no. 1, 2009, p. 28-40.

[3] Tounsi, M., Chaari, F., Abbes, Ms., Fakhfakh, T., Haddar, M., Effect of camshaft eccentricity and follower backlash on the dynamic behaviour flexible cam mechanism, Diagnostyka, vol. 2, no. 54, 2010 p. 3 - 9.

[4] Abu, R., Oladejo K.A., Oriolowo, K.T., Amedu, S., Development of a computer-based model for cam analysis, International Conference of Mechanical Engineering, Energy Technology and Management, Nigeria, 2016 , p. $571-589$.

[5] Naghipoor, J., Ahmadian, S.A., Ali, R.S, An improved regula falsi method for finding simple zeros of nonlinear equations, Applied Mathematical Sciences, vol. 2, no. 8, 2008, p. 381 -386.

[6] Desai, H.D., Patel, V.K., Computer aided kinematic and dynamic analysis of cam and follower, Proceedings of the World Congress on Engineering, London, U.K., vol. 2, 2010, p. 1-5.

[7] Mina, H., Homayoun, N., William, C., A teaching tool for design and analysis of cam and follower mechanisms, American Society for Engineering Education, 2008, p. 1-10.

[8] Oladejo, K.A, Adetan, D.A., Obayopo, S.O., Oke, A.O., Adio, S.A, A low-cost laboratory experimental model of the valve-operating cam-follower mechanism of an internal combustion engine, Journal of Sustainable Technology, vol. 4, no. 2, 2013, p. 1-9.

[9] Oladejo, K.A., Adekunle, N.O., Oriolowo, K.T., Adeniji, D.O., The dynamic analysis of cam and follower mechanisms, Nigerian Institute of Industrial Engineers, International Conference, Nigeria, 2015, p. 101-114.

[10] Abu, R., Oladejo, K.A., Adekanmbi, U.O., Development of a model for the beam analysis, International Conference of Mechanical Engineering, Energy Technology and Management, Nigeria, 2014, p. 562-578.

[11] Le, D., An efficient derivative-free method for solving nonlinear equations, Transactions on Mathematical Software, vol. 11, no. 3, 1985, p. 250-262.

[12] Demeulenaere, B., Schutter, J.D., Accurate realization of follower motions in high-speed cam-follower mechanisms, Proceedings of ISMA 2002, vol. 3, 2002, p. 1107-1116.

[13] Dass, H.K., Advance engineering mathematics, First Edition, Ed. S. Chand and Company Pvt. Ltd., New Delhi, 1988.

[14] Shigley, J.E., Uicker, J.J., Theory of machines and mechanism, Ed. McGraw-Hill, New York, 1980.

[15] Steven, C.C., Solution manual to accompany applied numerical method with matlab for engineers and scientist, Second Edition, ed. McGraw-Hill, 2000. 